\title{
Fluoroscopy-guided Umbilical Venous Catheter Placement in Infants with Congenital Heart Disease
}

\author{
Aaron G. DeWitt, MD, Jeffrey D. Zampi, MD, Janet E. Donohue, MPH, Sunkyung Yu, MS, and \\ Thomas R. Lloyd, MD \\ Department of Pediatrics and Communicable Diseases, Division of Pediatric Cardiology, University of Michigan, Ann \\ Arbor, Mich, USA
}

\section{A B S T R A C T}

Objective. The objective of this study was to (1) describe the technical aspects of fluoroscopy-guided umbilical venous catheter placement (FGUVCP); and (2) determine the procedural success rate, factors contributing to procedural failure, and risks of the procedure.

Background. Umbilical venous catheters are advantageous compared with femoral venous access, but can be difficult to place at the bedside.

Materials and Methods. This was a retrospective chart review from a single tertiary care referral institution.

Results. FGUVCP was successful in 138 of 180 patients (76.7\%) over a seven-year period. Patients in whom FGUVCP was successful were younger at the time of procedure compared with patients in whom FGUVCP was unsuccessful (median 18.2 vs. 22.2 hours, $P=.03$ ). The optimal age cutoff to predict FGUVCP success was 20 hours with a high positive predictive value $(82.4 \%)$ but low negative predictive value $(32.5 \%)$. No other variables were associated with procedural failure, though functional univentricular heart and older gestational age trended toward statistical significance. Median radiation time, contrast exposure, and blood loss were 3.2 minutes, $1 \mathrm{~mL}$, and $1 \mathrm{~mL}$, respectively. A total of 10 complications in 10 patients were associated with FGUVCP.

Conclusions. FGUVCP is a safe and highly successful way to obtain central venous access in neonates with congenital heart disease. Older age at the time of procedure is associated with procedural failure, but utilization of an age cutoff may not be clinically useful.

Key Words. Umbilical Vein; Catheterization; Ductus Venosus; Central Venous Access

\section{Introduction}

$\mathrm{M}$

any infants with congenital heart defects will require surgery in the neonatal period. Central venous access is useful in these patients for hemodynamic monitoring and medication administration both before and after surgery. While the typical routes of central venous access-the femoral, internal jugular, and subclavian veinsare available in this population, neonates have a unique route of access: the umbilical vein.

Work was performed at the University of Michigan C.S. Mott Children's Hospital.

This study was performed with funding from the Division of Pediatric Cardiology fellowship general research funds.
In fetal circulation, the umbilical vein transports oxygen rich blood from the placenta through the liver via the ductus venosus to the inferior vena cava. Postnatally, the umbilical vein maintains its connection to the central circulation, provided the ductus venosus remains open. ${ }^{1,2}$ Typically, umbilical venous catheters (UVCs) can be directly inserted into the umbilical stump and advanced into the right atrium via the ductus venosus at the bedside. Successful placement is typically confirmed by frontal chest/abdomen bedside radiographs with the tip of the catheter projecting above the right hemi-diaphragm (in patients with abdominal situs solitus).

When UVC placement is unsuccessful, it is usually because the catheter does not cross the 
ductus venosus and deflects into the portal circulation. ${ }^{3}$ Fortunately, UVC placement is successful about half of the time on first attempt using the standard technique of manual insertion. ${ }^{4,5}$ Various maneuvers have been described, such as the double catheter technique ${ }^{4,6-8}$ and posterior liver mobilization, ${ }^{5}$ which can increase the ultimate success rate to approximately $75 \%$. Other imaging modalities, such as ultrasound ${ }^{9-12}$ and electrocardiography, ${ }^{13}$ can help determine the location of the catheter tip, but do not increase success rates. Of course, if the ductus venosus closes prior to UVC placement, the procedure will be unsuccessful, but no bedside UVC placement technique currently described in the literature determines its patency.

UVCs are relatively safe in the short term as long as they are placed correctly.,14 In a recent review of neonates after congenital heart surgery from University of Michigan C.S. Mott Children's Hospital, Aiyagari et al. demonstrated superior safety of UVCs compared with femoral venous catheters and a high success rate for UVC placement. ${ }^{15}$ This review generated considerable interest in the techniques employed at our institution prompting a review of our practice. For over 20 years, pediatric cardiologists have been developing the technique of fluoroscopy-guided UVC placement (FGUVCP) in the cardiac catheterization laboratories at our institution. In this report, we describe this previously unpublished technique. Furthermore, by reviewing patient and procedural data from a substantial sample of neonates in whom FGUVCP was attempted, we describe our success rate, determine what factors contribute to procedural failure, and identify the risks of the procedure. In particular, we aim to understand how age at procedure impacts success rate, as age is the only truly modifiable factor.

\section{Materials and Methods}

\section{Study Description}

All patients in whom placement of an UVC was attempted in the University of Michigan Congenital Heart Center catheterization laboratories between November 1, 2005 and November 30, 2012 were included. Five attending physicians performed this procedure during this time period. The study was approved with a waiver of informed consent by our institutional review board.

Patients were identified by search of the catheterization laboratory logbooks and software systems (WITT Biomedical Calysto Series IV and Xper Information Management, Philips, Best,
The Netherlands) and procedural reports were reviewed. If review of the procedural $\log$ and report indicated that FGUVCP was attempted, the patient's UVC status on arrival to the catheterization laboratory was noted. If the patient had an appropriately placed UVC on arrival which was exchanged to facilitate a diagnostic or interventional procedure, the patient was excluded from the study. If the patient had failed bedside UVC placement, had prior successful bedside UVC placement but the UVC had been inadvertently withdrawn from the central circulation, or had not had bedside UVC placement attempted, further demographic and procedural data were collected. These included date and time of birth, gestational age, date and time of procedure, procedure length, cardiac diagnosis, periprocedure prostaglandin use, presence of heterotaxy syndrome, fluoroscopy time, iodinated contrast use, blood loss, additional procedures performed, complications of the procedure(s), result of angiogram (if applicable), and whether or not FGUVCP was successful.

Two cohorts were created for data analysis. The first cohort comprised all subjects who met inclusion criteria. This cohort was analyzed to determine FGUVCP success rate and to determine the effects of patient characteristics on that success rate. The second cohort was created to determine procedural data including fluoroscopy time, iodinated contrast dose, and procedural blood loss attributable to FGUVCP. It comprised only those subjects without an additional procedure (e.g., percutaneous balloon atrial septostomy) with two exceptions: (1) subjects who had umbilical artery catheter (UAC) replacement if it was dislodged by FGUVCP (as UAC dislodgement was considered a complication of FGUVCP); and (2) subjects in whom FGUVCP was unsuccessful and central venous access was attempted at another site.

\section{FGUVCP Technique}

Institutional practice is to attempt UVC placement at the bedside if an emergent intervention is not necessary. Most often, this is performed by the neonatal team in the neonatal intensive care unit for patients born at the C.S. Mott Children's Hospital or by the neonatal team at a referring institution. Less often, UVC placement occurs in our institution's pediatric cardiothoracic intensive care unit. The techniques used to enhance successful bedside placement (e.g., double catheter technique, posterior liver mobilization, etc.) are variable and dependent on the experience of the provider placing the catheter. 
When bedside UVC placement is unsuccessful, the catheter is withdrawn, secured at $5 \mathrm{~cm}$ in a "low-lying" position, and FGUVCP is scheduled if requested by the cardiac intensivist. After obtaining informed consent from the parents or guardians, the patient is brought to the catheterization laboratory and placed supine on the catheterization table. If no other procedures are deemed necessary, this procedure is performed without sedation or with light sedation.

After antiseptic preparation and sterile draping of the umbilical region, a new 5 French double- or triple-lumen UVC is prepared by flushing all lumens with heparinized saline. A $3 \mathrm{~mL}$ syringe filled with iodinated contrast is connected to a secondary lumen of the catheter and flush syringes are connected to the remaining catheter lumens. The existing low-lying UVC is removed and the new UVC is inserted into the umbilical vein. The new UVC is advanced until light resistance is met. A steerable 0.018-inch wire such as an angled Glidewire (Terumo Medical Corporation, Somerset, NJ, USA) or a Cope wire (Cook, Bloomington, IN, USA) is advanced into the primary lumen of the catheter and, under fluoroscopic guidance, the wire is advanced beyond the catheter tip.

In some cases, the wire will pass across the patent ductus venosus directly into the right atrium. If this occurs, the catheter is advanced over the wire and its tip is positioned at the cavoatrial junction. The wire is removed from the primary lumen and blood is withdrawn from all lumens and the lumens are then flushed. The UVC is then secured with suture and the procedure is complete.

In most cases, the wire does not gain immediate access to the right atrium and a small amount of contrast is injected through the secondary lumen. Biplane fluoroscopy/angiography is recommended as the ductus venosus takes a very posteriorly directed course, which is not readily visualized on frontal projection (Figure 1A,B). With the course of the ductus venosus visualized, the wire is used to cross into the right atrium.

In some cases, the ductus venosus is patent by contrast venography but is tortuous or of small caliber, and the UVC does not readily follow the wire into the right atrium. In these circumstances, a number of maneuvers can be attempted. One such maneuver is to serially dilate the tract with 3 French, 4 French, and 5 French dilators. Another useful maneuver is to replace the initial guidewire with a stiffer wire. Passing a 0.014-inch guidewire through an additional lumen of the UVC can also provide added "stiffness" and facilitate advancing

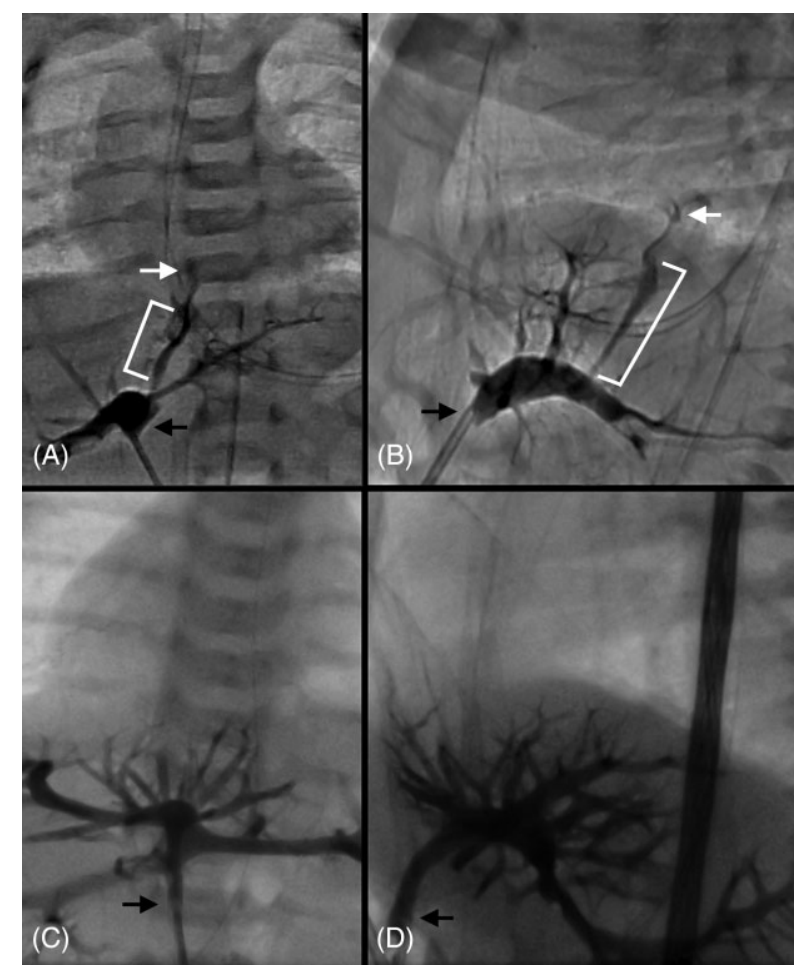

Figure 1. Umbilical vein angiography. Contrast is injected from the tip of the umbilical venous catheter (black arrows). In the upper two images, an open ductus venosus can be seen (white brackets) with a puff of contrast entering the right atrium (white arrows) in frontal (A) and lateral (B) projections. In the lower two images, the ductus venosus is closed and not identified in either frontal (C) or lateral (D) projections. Note the prominent opacification of the portal veins that is seen when the ductus venosus is closed ( $C$ and D) compared with when it is open (A and $B$ ).

the catheter. These maneuvers can be used singly or in combination. Whatever wires are selected must have a very soft and pliable tip in order to avoid perforation of the hepatic or cardiac structures.

If there is no evidence of a patent ductus venosus by angiography (Figure $1 \mathrm{C}, \mathrm{D})$, the procedure is abandoned. Even if the ductus venosus is in spasm or functionally closed, there is no "roadmap" for advancing a guidewire. Similarly, if a wire cannot traverse the ductus venosus, the procedure is also abandoned. Central venous access at another site is often attempted if the referring physician requests and if work flow in the catheterization laboratory permits.

\section{Statistical Analysis}

Demographic, clinical, and procedural characteristics were presented as frequency (\%) for categorical variables and median (interquartile range) 
Table 1. Comparison of Patient and Clinical Characteristics for Neonates by FGUVCP Result $(N=180)$

\begin{tabular}{|c|c|c|c|c|}
\hline & \multirow{2}{*}{$\begin{array}{l}\text { Overall } \\
(N=180)\end{array}$} & \multicolumn{2}{|l|}{ FGUVCP success } & \multirow[b]{2}{*}{$P$ value* } \\
\hline & & $\begin{array}{l}\text { Yes } \\
(N=138)\end{array}$ & $\begin{array}{l}\text { No } \\
(N=42)\end{array}$ & \\
\hline Age at procedure, $\mathrm{h}$ & $19.3(8.3-32.1)$ & $18.2(7.5-29.0)$ & $22.2(14.6-45.8)$ & .03 \\
\hline$<20$ & & 75 (54.3) & $16(38.1)$ & .02 \\
\hline$\geq 20$ & & $54(39.1)$ & $26(61.9)$ & \\
\hline Missing & & $9(6.5)$ & $0(0.0)$ & \\
\hline$<24$ & & $89(64.5)$ & $23(54.8)$ & .09 \\
\hline$\geq 24$ & & $40(29.0)$ & $19(45.2)$ & \\
\hline Missing & & $9(6.5)$ & $0(0.0)$ & \\
\hline$<36$ & & $106(76.8)$ & $29(69.0)$ & .07 \\
\hline$\geq 36$ & & $23(16.7)$ & $13(31.0)$ & \\
\hline Missing & & $9(6.5)$ & $0(0.0)$ & \\
\hline Male sex & $111(61.7)$ & $84(60.9)$ & 27 (64.3) & .69 \\
\hline Gestational age, wk & $39(38-39.6)$ & 39 (37.9-39.4) & $39.2(38.2-39.7)$ & .07 \\
\hline Preterm (less than $37 \mathrm{wk}$ ) & & $23(16.7)$ & $4(9.5)$ & .27 \\
\hline Birth weight, kg & $3.2(2.95-3.5)$ & $3.2(2.9-3.5)$ & $3.1(2.9-3.4)$ & .97 \\
\hline \multicolumn{5}{|l|}{ Cardiac diagnosis } \\
\hline Functional univentricular heart & $86(47.8)$ & $61(44.2)$ & $25(59.5)$ & $.08^{\dagger}$ \\
\hline Hypoplastic left heart syndrome & $48(26.7)$ & $33(23.9)$ & $15(35.7)$ & \\
\hline Pulmonary atresia with intact ventricular septum & $9(5.0)$ & $8(5.8)$ & $1(2.4)$ & \\
\hline Tricuspid atresia & $8(4.4)$ & $7(5.1)$ & $1(2.4)$ & \\
\hline Unbalanced atrioventricular septal defect & $11(6.1)$ & $7(5.1)$ & $4(9.5)$ & \\
\hline Other & $10(5.6)$ & $6(4.3)$ & $4(9.5)$ & \\
\hline Transposition physiology & $38(21.1)$ & $33(23.9)$ & $5(11.9)$ & \\
\hline Left heart obstructive lesion & $21(11.7)$ & $17(12.3)$ & $4(9.5)$ & \\
\hline Right heart obstructive lesion & $19(10.6)$ & $13(9.4)$ & $6(14.3)$ & \\
\hline Balanced atrioventricular septal defect & $2(1.1)$ & $2(1.4)$ & $0(0.0)$ & \\
\hline Other & $14(7.8)$ & $12(8.7)$ & $2(4.8)$ & \\
\hline Heterotaxy syndrome & $15(8.3)$ & $9(6.5)$ & $6(14.3)$ & .12 \\
\hline Receiving prostaglandin infusion & $158(87.8)$ & $121(87.7)$ & $37(88.1)$ & .94 \\
\hline
\end{tabular}

Data are presented as $N$ (\%) for categorical variables and median (25th-75th percentiles) for continuous variables.

${ }^{*} P$ value from chi-square test or Fisher's exact test, as appropriate, for categorical variables, and Wilcoxon rank-sum test for continuous variables.

tComparison was made between functional univentricular heart vs. all others, and $P$ value was from chi-square test.

FGUVCP, fluoroscopy-guided umbilical venous catheter placement.

for continuous variables. Group comparisons between successful and unsuccessful FGUVCP were made using chi-square tests or Fisher's exact tests, as appropriate, for categorical variables, and Wilcoxon rank-sum tests for continuous variables. A receiver operating characteristic (ROC) curve was generated to determine an optimal age cutoff for significant discrimination of FGUVCP success. Odds ratios (ORs) with 95\% confidence intervals (CIs) were also estimated using logistic regression to evaluate the effect of age at procedure on FGUVCP using the optimal cutoff obtained from the ROC curve. All analyses were performed using SAS version 9.3 (SAS Institute, Cary, NC, USA), with statistical significance set at $P$ value less than 0.05 using two-sided tests.

\section{Results}

Inclusion criteria were met by 180 patients. Among those, bedside placement had been unsuccessful in 163 patients $(90.6 \%)$. The first attempt at UVC placement was in the catheterization laboratory in 11 patients $(6.1 \%)$, and in 6 patients
(3.3\%) FGUVCP was utilized after a previously well-positioned bedside UVC was noted to have migrated out of the central circulation. Patient demographics and clinical characteristics are shown in Table 1. Additional procedures beyond FGUVCP were performed in 110 patients, 22 of whom only had central venous catheter placement at another site.

Overall, FGUVCP was successful in 138 patients $(76.7 \%)$ and unsuccessful in 42 patients (23.3\%). In the unsuccessful group, contrast venography demonstrated patency of the ductus venosus in $31 \%$ of patients (13/42). Among the patients in whom initial bedside placement was unsuccessful $(N=163), 122(74.8 \%)$ patients had successful FGUVCP.

Patients in whom FGUVCP was successful were younger than those in whom it was unsuccessful (median 18.2 hours vs. 22.2 hours, $P=.03$ ) (Table 1). Precise data needed to determine age at procedure were missing in nine patients. The number of patients with successful and unsuccessful FGUVCP by age at procedure is illustrated in Figure 2. No other patient characteristics were 
found to have a significant association with FGUVCP results, though functional univentricular heart $(P=.08)$ and older gestational age $(P=$ $.07)$ trended toward association with FGUVCP failure.

Utilizing the ROC curve, the optimal cutoff for age at the time of procedure to identify the patients with procedural success was 20 hours (area under the curve $[\mathrm{AUC}]=0.61$ ) with sensitivity of $58.1 \%$, specificity of $61.9 \%$, positive predictive value of $82.4 \%$, and negative predictive value of $32.5 \%$. Patients less than 20 hours of age at procedure were more likely to have successful FGUVCP than those with age of 20 hours or greater $(P=.02)$. Age cutoff of 24 and 36 hours did not reach statistical significance $(P=.09$ and .07 , respectively [Table 1]). In multivariate analysis, when adjusting for cardiac diagnosis, age less than 20 hours at the time of procedure remained significantly associated with FGUVCP success (OR 2.36, 95\% CI 1.14, 4.86; $P=.02$ ) (Table 2).

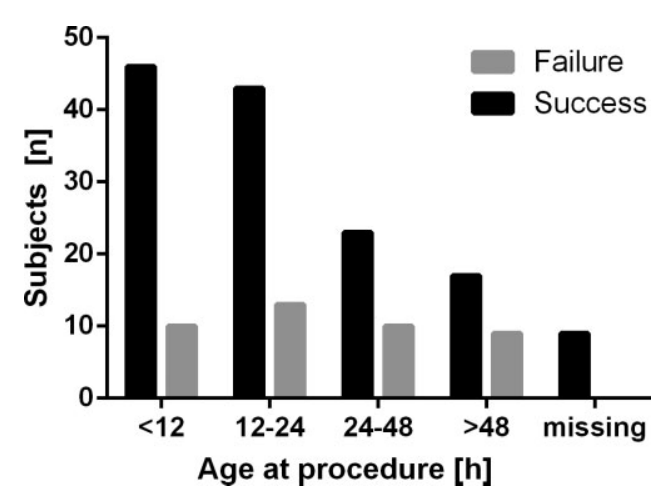

Figure 2. Number of patients with procedural success and failure after attempted fluoroscopy-guided umbilical venous catheter placement by age at procedure. Success rates were $82.1 \%$ for patients less than 12 hours of age, $76.8 \%$ for patients 12-24 hours of age, $69.7 \%$ for patients $24-48$ hours of age, and $65.4 \%$ for patients older than 48 hours of age. Age at procedure data were missing in nine patients, all of whom had successful umbilical venous catheter placement.
Procedural data are shown in Table 3 for the second cohort described above. A total of 114 patients only had central venous line placement, 92 had FGUVCP alone, and 22 had failed FGUVCP and central venous access at a secondary site. The median procedure time was 52.0 minutes when only FGUVCP was attempted and 78.4 minutes when FGUVCP failed and a central venous catheter was placed at another site. Similarly, fluoroscopy time, contrast dose, and blood loss were all low when only FGUVCP was attempted and slightly higher when FGUVCP failed and a central venous catheter was placed at another site.

In the entire 180-patient cohort, there were a total of 17 complications. In seven patients, the complication was attributed to a non-FGUVCP portion of the procedure (e.g., bradycardia with balloon atrial septostomy). Of the remaining 10 complications attributable to FGUVCP, seven patients had dislodgement of the umbilical arterial catheter associated with transportation to the catheterization laboratory or during the procedure itself. Two patients had transient heart rhythm disturbances (one with hemodynamically insignificant atrial flutter which self-resolved and one with complete heart block requiring atropine). Finally, in one patient, cardiopulmonary resuscitation was required. According to the report, the patient "started to cry and appeared to have a breath holding spell" with subsequent respiratory arrest and ventricular fibrillation requiring less than 1 minute of compressions. This occurred after the line was placed and while it was being secured with suture.

\section{Discussion}

In this population of neonates with congenital heart disease (CHD), FGUVCP is highly successful $(76.7 \%)$, which is higher than the $50-75 \%$ that is reported in the literature for bedside placement techniques. ${ }^{4-8}$ This is an invalid comparison as the

Table 2. Odds of Successful FGUVCP $(N=180)$

\begin{tabular}{|c|c|c|c|c|c|c|}
\hline & \multicolumn{3}{|c|}{ Unadjusted } & \multicolumn{3}{|c|}{ Adjusted } \\
\hline & OR & $95 \% \mathrm{Cl}$ & $P$ value ${ }^{*}$ & AOR & $95 \% \mathrm{Cl}$ & $P$ value $^{\dagger}$ \\
\hline \multicolumn{7}{|l|}{ Age at procedure, $\mathrm{h}$} \\
\hline$<20$ & 2.26 & $1.11,4.61$ & .02 & 2.36 & $1.14,4.86$ & .02 \\
\hline$\geq 20$ & Ref & & & Ref & & \\
\hline \multicolumn{7}{|l|}{ Cardiac diagnosis } \\
\hline Functional univentricular heart & 0.54 & $0.27,1.09$ & .08 & 0.53 & $0.26,1.08$ & .08 \\
\hline All others & Ref & & & Ref & & \\
\hline
\end{tabular}


Table 3. Procedure Characteristics of Neonates Who Had FGUVCP

\begin{tabular}{|c|c|c|}
\hline & $\begin{array}{l}\text { FGUVCP only } \\
\text { and no other } \\
\text { procedures done } \\
(N=92)\end{array}$ & $\begin{array}{l}\text { Unsuccessful } \\
\text { FGUVCP, } \\
\text { alternative central } \\
\text { venous access } \\
\text { obtained } \\
(N=22)\end{array}$ \\
\hline $\begin{array}{l}\text { Procedure length, min } \\
\text { Fluoroscopy time, min }\end{array}$ & $52.0(39.9-72.8)$ & $78.4(58.1-90)$ \\
\hline Total & $3.2(1.5-7.8)$ & $5.6(2.5-8.4)$ \\
\hline AP & $3.0(1.1-6.4)$ & $4.4(2.2-6.9)$ \\
\hline LAT & $0.0(0.0-0.9)$ & $0.3(0.0-1.2)$ \\
\hline Contrast used, $\mathrm{mL}$ & $1(0-2)$ & $2(2-3)$ \\
\hline 0 & $28(30.4)$ & $0(0.0)$ \\
\hline $0-2$ & $30(32.6)$ & $3(13.6)$ \\
\hline 2 & $20(21.7)$ & $11(50.0)$ \\
\hline$\geq 3$ & $14(15.2)$ & $8(36.4)$ \\
\hline Blood loss, $\mathrm{mL}$ & $1(1-2)$ & $3(2-5)$ \\
\hline$<1$ & 15 (16.3) & $0(0.0)$ \\
\hline 1 & $36(39.1)$ & $2(9.1)$ \\
\hline 2 & $19(20.7)$ & $5(22.7)$ \\
\hline$\geq 3$ & $21(22.8)$ & $15(68.2)$ \\
\hline Missing & $1(1.1)$ & \\
\hline Complication & $8(8.7)$ & $2(9.1)$ \\
\hline
\end{tabular}

Data are presented as $N(\%)$ or median (25th-75th percentiles), as appropriate.

FGUVCP, fluoroscopy-guided umbilical venous catheter placement.

true measure of the impact of FGUVCP is the nearly $75 \%$ success rate for the 163 patients in whom initial bedside placement was unsuccessful.

Unfortunately, the institutional success rate of standard bedside insertion in CHD patients is unknown. When bedside UVC placement is difficult, providers at our institution may opt to abandon the procedure in favor of FGUVCP. This selection bias would cause the FGUVCP success rates to be artificially inflated. On the other hand, it is conceivable that our patient population may have other characteristics (term gestational age, complex CHD, etc.) that may make bedside UVC placement more difficult. For instance, in the study by Al-Essa et al., when UVC placement was attempted at $<24$ hours of life, the procedure was successful in $17 / 18(94.4 \%)$ of neonates $<38$ weeks gestational age but only successful in $2 / 5(40.0 \%)$ of neonates $\geq 38$ weeks gestational age. ${ }^{7}$ In our study's cohort, $75 \%$ of subjects had a gestational age of $\geq 38$ weeks conceivably making UVC placement more difficult. Although a prospective study would be necessary to truly delineate the degree to which FGUVCP increases UVC utilization, a study from our institution found that when UVC placement was attempted in a cohort of single ventricle patients, it was successful $83 \%$ of the time. ${ }^{15}$ Thus, utilizing standard bedside placement techniques and FGUVCP, the institutional success rate for term patients with functional univentricu- lar hearts, a population in which UVC placement may be more difficult, is higher than the $75 \%$ success rate that is reported in the literature for all (but predominately premature) neonates. ${ }^{4-8}$

The importance of increasing UVC utilization rates in neonates requiring congenital heart surgery is clear. Many patients will require central venous access, particularly femoral venous access, for a wide range of future surgical and/or percutaneous interventions. The previously cited study by Aiyagari et al. of neonates with single ventricle heart disease serves as example to illustrate this importance: the authors found lower rates of both femoral venous thrombosis and iliofemoral venous occlusion when initial line placement was a UVC compared with those with femoral central venous catheter. ${ }^{15}$ Given the increased absolute risk of venous thrombotic disease in patients with femoral lines in that study, the number of UVCs that need to be placed in order to prevent one case of femoral venous thrombosis and one case of iliofemoral occlusion is three and four, respectively. ${ }^{15}$ Furthermore, while intravenous heparin infusion has been shown to prevent thrombosis of central venous catheters in children, ${ }^{16}$ this has been shown not to be the case in infants after cardiac surgery. ${ }^{17}$ Therefore, initial UVC placement is an attractive option for our patients in order to increase the likelihood of central venous patency.

Not unexpectedly, older age at the time of FGUVCP was associated with an unsuccessful procedure. While statistically significant, the clinical significance of this finding is not clear. We recommend that age should not be used as a cutoff for deciding who should and who should not have FGUVCP attempted. Age at procedure was available in 171 subjects and while those younger than our optimal age cutoff of 20 hours did have successful FGUVCP $82.4 \%$ of the time (75 of 91), those at that cutoff or older still had successful FGUVCP $67.5 \%$ of the time (54 of 80 ). Further, the AUC for the ROC curve was low (0.61), which is close to that of the line of no discrimination $(\mathrm{AUC}=0.5)$. Finally, although one should not base clinical decisions based on the extreme cases, in this series we documented ductus venosus closure in an infant less than 5 hours old and successful FGUVCP after 5 days of life.

We conclude, however, that these results should impact institutional approaches to FGUVCP. Given the higher success rates associated with the 20-hour age cutoff, our policy is to encourage FGUVCP within the first 24 hours of life, irrespective of day of the week. This may not be fea- 
sible at all institutions, and the decision to devote the resources needed to offer FGUVCP during off-hours merits careful consideration.

Looking at other factors besides age, having a functionally univentricular heart seemed to be associated with FGUVCP being unsuccessful though this association failed to reach statistical significance. Again, the clinical significance is not clear as this finding was surprising and we do not have a satisfactory anatomical or physiological explanation. Older gestational age also seemed to be associated with FGUVCP failure. This finding was less surprising. Because the ductus arteriosus tends to persist longer in younger gestational age neonates, it is reasonable to hypothesize the ductus venosus may as well. However, the magnitude of the difference seen between successful and failed FGUVCP (median gestational age of 39 and 39.2 weeks, respectively) was not clinically significant. We expected heterotaxy syndrome to be associated with procedural failure, but the number of patients with heterotaxy in our study was too small to reach statistical significance. Anecdotally, patients with heterotaxy syndrome can have an abnormally situated and tortuous ductus venosus making FGUVCP difficult even if the ductus venosus is patent.

We were unable to demonstrate an association between prostaglandin infusion and ductus venosus patency. This is likely because so many of our patients were on prostaglandins at the time of their procedure. The question of whether prostaglandin has a similar effect on the ductus venosus as it does the ductus arteriosus is interesting and a topic for future research.

While only $25 \%$ of patients in whom bedside placement of UVCs was unsuccessful also had failed FGUVCP, it would be advantageous if we could identify this group prospectively, Identifying ductus venosus closure at the bedside could lead to fewer FGUVCP failures decreasing resource utilization. Venous contrast echocardiography could potentially aid in this. With the use of agitated saline, venous contrast echocardiography has been shown to identify atrial level shunts, ${ }^{18}$ pulmonary arteriovenous malformations, ${ }^{19}$ and left superior vena cava. ${ }^{20} \mathrm{It}$ is conceivable that injecting agitated saline into a "low-lying" UVC would show opacification of the atrial chamber(s) with microbubbles if the ductus venosus was still patent, but the contrast would be filtered in the portal capillary network resulting in no opacification of the heart chambers if the ductus venosus was closed. This has the potential for future research.
There are some important practical considerations when performing FGUVCP. In patients in whom bedside placement is unsuccessful, the "lowlying" UVC is often the only source of venous access prior to FGUVCP. This position would not be considered central, so high doses of adrenergic agents and high concentrations of parenteral nutrition should be avoided. Further, if it is anticipated that intravenous medication will be necessary during the procedure (e.g., sedation, inotropic support), a second site of venous access should be obtained since the process of FGUVCP involves removing the original catheter. In one patient who was excluded from analysis, FGUVCP was never attempted because there was no other venous access and he was requiring frequent adenosine doses for supraventricular tachycardia.

Another important consideration is how to image the ductus venosus. We found that the amount of lateral fluoroscopy exposure was low. Indeed, in the 92 patients in whom only FGUVCP was attempted, $52(57 \%)$ patients had 0 minute of lateral fluoroscopy exposure. However, the importance of the lateral imaging to visualize the ductus venosus, which was described as early as $1969,{ }^{21}$ cannot be overemphasized. This is especially true in difficult cases as the ductus venosus takes a very posterior course, which is difficult to appreciate by frontal imaging.

Finally, we have demonstrated that this is a safe procedure with minimal blood loss and exposure to intravenous contrast and radiation. One major complication occurred after the FGUVCP had been completed. The causal relationship of securing the catheter to the respiratory arrest is unclear. Two patients had transient arrhythmias which is a known risk of intraatrial wire and catheter manipulation. The other minor complications involved inadvertent dislodgement of umbilical arterial catheters. Generally, our neonatal critical care team colleagues do not secure the umbilical catheters with suture. Therefore, dislodgement of the umbilical arterial catheter could potentially be avoided if they are sutured to the umbilical stump prior to transport.

Limitations of this study include its retrospective nature. A prospective study would be necessary to truly understand the individual and combined success rate of bedside UVC placement and FGUVCP. Another limitation is the heterogeneity of cardiac diagnoses in this sample. The number of subjects in each anatomic subgroup was small. Lastly, there is individual variability among cardiac interventionalists who perform this proce- 
dure. An institutional protocol could improve case-by-case variability.

\section{Conclusion}

FGUVCP is a safe and relatively simple procedure that can be performed readily in a pediatric cardiac catheterization laboratory for patients in whom bedside UVC placement was unsuccessful. In patients with CHD, older age at the time of procedure is associated with FGUVCP failure. However, we do not recommend withholding FGUVCP from older neonates.

\section{Acknowledgements}

We would like to acknowledge the efforts over the years of the pediatric catheterization laboratory physicians, nurses, and technicians at the University of Michigan C.S. Mott Children's Hospital Congenital Heart Center. Their dedication to this procedure has allowed hundreds of infants with congenital heart disease to have appropriately placed umbilical venous catheters.

Corresponding Author: Thomas R. Lloyd, MD, Department of Pediatrics and Communicable Diseases, Division of Pediatric Cardiology, University of Michigan, 1540 East Hospital Drive, Ann Arbor, MI 481094204, USA. Tel: 734-936-9114; Fax: 734-936-9470; E-mail: 1loydt@med.umich.edu

Conflict of interest: The authors have no potential conflicts of interest to disclose.

Accepted in final form: October 02, 2014.

\section{References}

1 Oestreich A. Umbilical vein catheterizationAppropriate and inappropriate placement. Pediatr Radiol. 2010;40:1941-1949.

2 Kiserud T. The ductus venosus. Semin Perinatol. 2001;25:11-20.

3 Sanders CF. The placement of the umbilical venous catheter in the newborn and its relationship to the anatomy of the umbilical vein, ductus venosus and portal venous system. Clin Radiol. 1978;29:303308.

4 Haase R, Hein M, Thäle V, Vilser C, Merkel N. Umbilical venous catheters-analysis of malpositioning over a 10-year period. Z Geburtshilfe Neonatol. 2011;215:18-22.

5 Pennaforte T, Klosowski S, Alexandre C, Ghesquière J, Rakza T, Storme L. Increased success rate in umbilical venous catheter positioning by posterior liver mobilization. Arch Pediatr. 2010;17: 1440-1444.
6 Mandel D, Mimouni F, Littner Y, Dollberg S. Double catheter technique for misdirected umbilical vein catheter. 7 Pediatr. 2001;139:591592.

7 Al-Essa M, Rashwan N, Devarajan L. Doublecatheter technique for the proper insertion of umbilical venous catheters in newborns. Med Princ Pract. 2005;14:98-101.

8 Davies $M$, Cartwright D. Insertion of umbilical venous catheters past the ductus venosus using the double catheter technique. Arch Dis Child Fetal Neonatal Ed. 1998;78:234.

9 Simanovsky N, Ofek-Shlomai N, Rozovsky K, Ergaz-Shaltiel Z, Hiller N, Bar-Oz B. Umbilical venous catheter position: evaluation by ultrasound. Eur Radiol. 2011;9:1882-1886.

10 Ades A, Sable C, Cummings S, Cross R, Markle B, Martin G. Echocardiographic evaluation of umbilical venous catheter placement. $f$ Perinatol. 2003;23:24-28.

11 George L, Waldman J, Cohen M, et al. Umbilical vascular catheters: localization by two-dimensional echocardio/aortography. Pediatr Cardiol. 1982;2: 237-243.

12 Greenberg M, Movahed H, Peterson B, Bejar R. Placement of umbilical venous catheters with use of bedside real-time ultrasonography. $f$ Pediatr. 1995;126:633-635.

13 Tsui B, Richards GJ, Van Aerde J. Umbilical vein catheterization under electrocardiogram guidance. Paediatr Anaesth. 2005;15:297-300.

14 Loisel D, Smith M, MacDonald M, Martin G. Intravenous access in newborn infants: impact of extended umbilical venous catheter use on requirement for peripheral venous lines. 7 Perinatol. 1996;16:461-466.

15 Aiyagari R, Song JY, Donohue JE, Yu S, Gaies M. Central venous catheter-associated complications in infants with single ventricle: comparison of umbili$\mathrm{cal}$ and femoral venous access routes. Pediatr Crit Care Med. 2012;13:549-553.

16 Bracho-Blanchet E, Cortes-Sauza J, Davila-Perez R, Lezama-Del V, Villalobos-Alfaro C, NietoZermeño J. Usefulness of intravenous heparin to prevent thrombosis of central venous catheter in children. Cir Cir. 2010;78:423-429.

17 Schroeder AR, Axelrod DM, Silverman NH, Rubesova E, Merkel E, Roth SJ. A continuous heparin infusion does not prevent catheter-related thrombosis in infants after cardiac surgery. Pediatr Crit Care Med. 2010;11:489-495.

18 Konstadt SN, Louie EK, Black S, et al. Intraoperative detection of patent foramen ovale by transesophageal echocardiography. Anesthesiology. 1991;74:212-216.

19 Barzilai B, Waggoner AD, Spessert C, Picus D, Goodenberger D. Two-dimensional contrast echocardiography in the detection and follow-up of 
congenital pulmonary arteriovenous malformations. Am 7 Cardiol. 1991;68:1507-1510.

20 Palinkas A, Nagy E, Forster T, Nagy E, Varga A. A case of absent right and persistent left superior vena cava. Cardiovasc Ultrasound. 2006;4:6-9.
21 Baker DH, Berdon WE, James LS. Proper localization of umbilical arterial and venous catheters by lateral roentgenograms. Pediatrics. 1969;43:34-39. 